\title{
ENCEFALOPATIA HEPÁTICA: ESTUDO DE 50 CASOS
}

\author{
LUIZ ANTONIO DE LIMA RESENDE* \\ JOSE GERALDO SPECIALI**
}

Os conhecimentos em hepatologia remontam à Antigüidade. Os babilônios (cerca de 2000 a.C.) nomeavam o fígado pelo mesmo termo usado para alma ou estado de espírito. Os chineses (Nanquim, 1000 a.C.) consideravam o fígado armazenador do sangue, que continha a alma. Hipócrates (460 a 370 a.C.) descreveu paciente com hepatite "que latia como um cão, não podia ser contido e dizia coisas que não podiam ser compreendidas". Eristratus de Alexandria, em 300 a.C., teria associado o fígado de consistência pétrea à hidropsia hepática. Em 751 d.C. o Papa Zacarias escreveu carta a São Bonifácio, Arcebispo de Mainz, relatando casos de hepatite. Laënnec (1781 a 1826) empregou o termo cirrose, derivado do grego, kirrhos, que significa marron-amarelado. Em 1793 Matthew Baillie reconheceu a associação entre alcoolismo e cirrose (e talvez Vesalius, 1514/1564), a despeito da existència de hieróglifos de cerca de 1500 a.C. documentando o conhecimento egípcio sobre alguns dos males do alcoolismo (Fig. 1). Em 1866 Leyden usou o termo "colemia" atribuindo a encefalopatia a acúmulo de sais biliares no sangue. Frerichs, pai da hepatologia moderna, relatou: "Ocorreram-me casos nos quais pacientes que vinham sofrendo de cirrose por longo período passaram subitamente a apresentar uma série de sintomas mórbidos que são diferentes daqueles da doença. Tornaram-se inconscientes e presas de delírio agitado, do qual passaram para coma profundo, morrendo neste estado" 1, 14. Linde natural entre a insuficiência hepática e a morte, comum em cirróticos e de evolução imprevisivel, freqüentemente fatal, é a encefalopatia hepática condição grave e perigosa. Cumpre diagnosticá-la prontamente, para tratamento adequado e melhora da sobrevida.

No presente trabalho analisamos os aspectos clínicos, laboratoriais e anátomo-patológicos em 50 pacientes atendidos no Hospital das Clinicas da Faculdave de Medicina de Ribeirão Preto a partir de 1959.

Trabalho realizado no Departamento de Neuropsiquiatria e Psicologia Médica do Hospital das Clínicas da Faculdade de Medicina de Ribeirão Preto da Universidade de São Paulo: * Aluno de Pós-Graduação; ** Professor Assistente-Doutor. Agradecimento - Os autores agradecem ao Dr. Ulysses Meneghelli, Professor Adjunto da Disciplina de Gastroenterologia do Departamento de Clínica Médica da mesma Faculdade, pela grande colaboração prestada. 


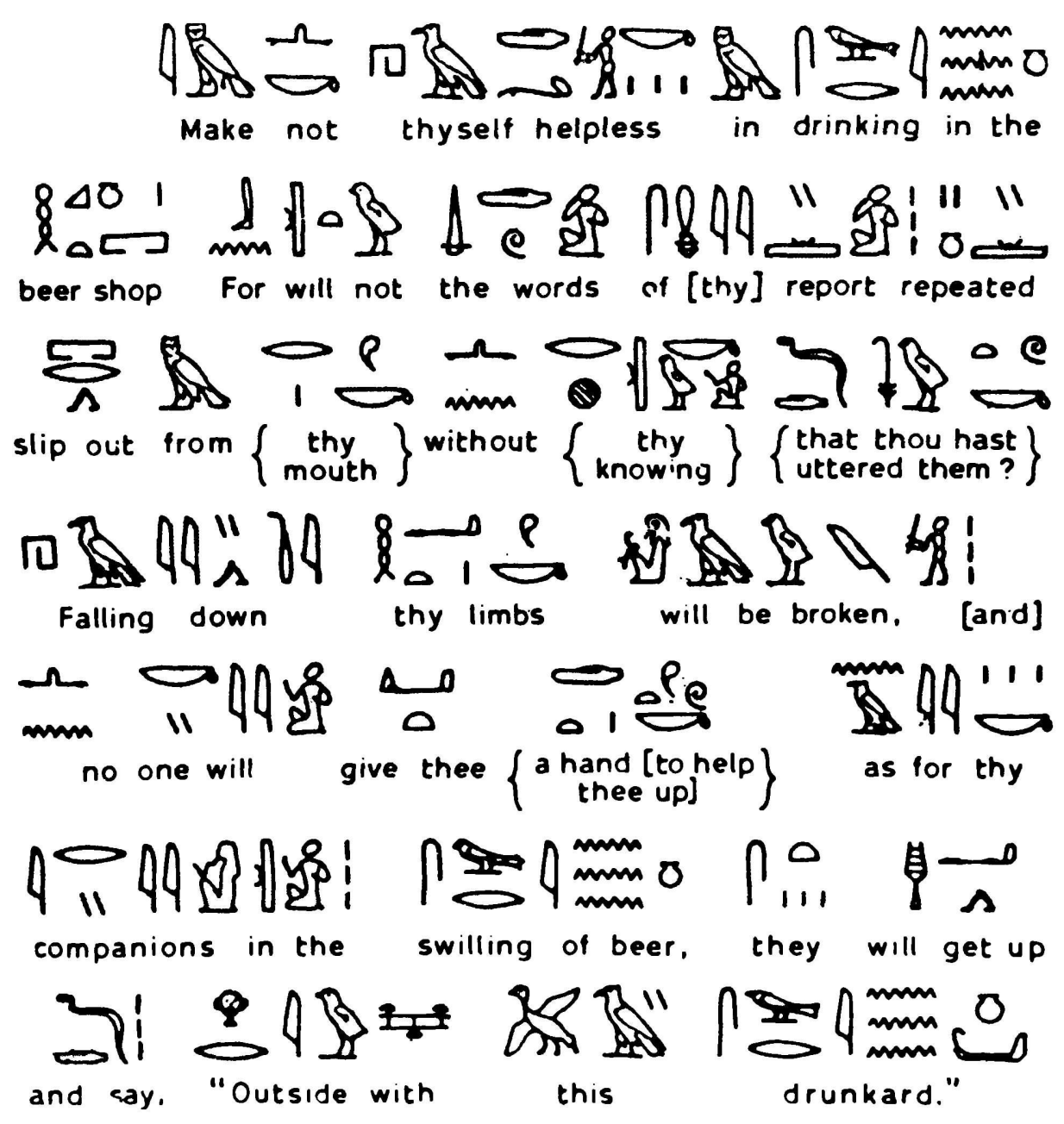

Frig. 1 - Hieróglifos de cerca de 1.500 a.C., provavelmente da época do fará Tutmosis III (XVIII Dinastia): Não bebas cerveja no bar até ficar incapaz de tão bebado, porque nepetirás coisas que te escaparão da boca sem que te des conta que as disseste. Caindo ao chão, quebrarás bnacos e pernas e ninguém te estenderd a mão para te ajudar, pois teus companheiros de bebedeira levantarão $e$ dirão "fora com este bebado". In: $L$. Schiff - Diseases of the Liver. Ed. 4. Lippincott, Philadelphia, 1975 (reproducáo autorizada).

\section{MATERIAL E METODOS}

Foi realizado estudo retrospectivo dos prontuários de 50 pacientes atendidos no Hospital das Clínicas da Faculdade de Medicina de Ribeirão Preto a partir de 1959 com quadros de encefalopatia hepática. Os diagnósticos das hepatopatias foram feitos nos 50 pacientes com base em critérios clínicos, laboratoriais e anátomo-patológicos, incluindo biópsia hepática ( 8 casos) e autópsia (20 casos). Um paciente, com hipótese diagnóstica de calazar, não teve o diagnóstico confirmado. Os diagnósticos de coma e pré-coma hepático foram estabelecidos com base em critérios clínicos e laboratoriais; entre estes, enfatizamos a gasometria arterial.

\section{RESULTADOS}

Nossa casuística inclui 27 pacientes do sexo masculino e 23 do sexo feminino, sendo 5 mulatos, 6 pretos e 39 brancos, com idades entre 1 ano e 8 meses e 74 anos. Na tabela 1 relacionamos nome, sexo, raç, idade, patologia hepática a a evoluçáo do caso. 


\begin{tabular}{|c|c|c|c|c|c|}
\hline Nome & Sexo & Raca & Idade & Patologia hepática & Evolução \\
\hline $\mathbf{A A}$ & $\mathbf{m}$ & b & 37 & Cirrose & $\mathbf{R}$ \\
\hline FSA & $\mathbf{f}$ & $\mathbf{b}$ & 57 & Cirrose & $\mathbf{0}$ \\
\hline ABSA & $\mathbf{I}$ & b & 28 & Cirrose & 0 \\
\hline EAB & $\mathbf{P}$ & $\mathbf{b}$ & 44 & Hepatite & $\mathbf{0}$ \\
\hline JRS & $\mathbf{m}$ & $\mathbf{b}$ & 28 & Hepatite & O \\
\hline GCS & $\mathbf{m}$ & $\mathbf{p}$ & 50 & Sarcoma de células Küpffer & 0 \\
\hline JBF & $\mathbf{m}$ & b & 44 & Cirrose & $\mathbf{0}$ \\
\hline NAS & $\mathbf{I}$ & $\mathbf{b}$ & 19 & Hepatite & 0 \\
\hline MJA & $\mathbf{f}$ & $\mathbf{b}$ & $20 \mathrm{~m}$ & Hepatite & $\mathbf{0}$ \\
\hline AJA & $\mathbf{m}$ & $\mathbf{p}$ & 11 & Hepatite & $\mathbf{O}$ \\
\hline CMS & $\mathbf{I}$ & $\mathbf{b}$ & 38 & Hepatite & $\mathbf{O}$ \\
\hline BMnN & $\mathbf{m}$ & $\mathbf{b}$ & 39 & Cirrose & 0 \\
\hline JCS & $\mathbf{m}$ & $\mathbf{m}$ & 20 & Cirrose & $\mathbf{O}$ \\
\hline GRP & $\mathbf{m}$ & $\mathbf{b}$ & 36 & Necrose tóxica (Cirrose) & $\mathbf{0}$ \\
\hline GAL & $\mathbf{f}$ & $\mathbf{m}$ & 19 & Cirrose & $\mathbf{O}$ \\
\hline MLM & $\mathbf{f}$ & $\mathbf{b}$ & 17 & Cirrose & $\mathbf{O}$ \\
\hline TFV & $\mathbf{P}$ & $\mathbf{b}$ & 2 & Hepatite & $\mathrm{O}$ \\
\hline EOS & $\mathbf{f}$ & $\mathbf{m}$ & 48 & Cirrose & $\mathbf{R}$ \\
\hline MSG & $\mathbf{f}$ & $\mathbf{p}$ & 43 & Esquistossomose & O \\
\hline LFP & $\mathbf{I}$ & b & 14 & Cirrose & $\mathbf{O}$ \\
\hline GL & $\mathbf{m}$ & $\mathbf{p}$ & 39 & Hepatite & $\mathbf{R}$ \\
\hline GP & $\mathbf{m}$ & $\mathbf{b}$ & 58 & Cirrose & 0 \\
\hline IG & $\mathbf{f}$ & $\mathbf{b}$ & 50 & Cirrose e esquistossomose & 0 \\
\hline BH & $\mathbf{f}$ & $\mathbf{b}$ & $\mathbf{5 5}$ & Cirrose & 0 \\
\hline AMN & f & $\mathbf{b}$ & 10 & Cirrose & 0 \\
\hline NRS & $\mathbf{P}$ & $\mathbf{m}$ & 58 & Cirrose & 0 \\
\hline ORS & $\mathbf{P}$ & $\mathbf{b}$ & 57 & Cirrose & 0 \\
\hline OBG & $\mathbf{m}$ & $\mathbf{b}$ & 48 & Hepatite & $\mathbf{R}$ \\
\hline CRO & $\mathbf{m}$ & b & 33 & Cirrose & $\mathbf{R}$ \\
\hline LPC & $\mathbf{2}$ & $\mathbf{b}$ & 74 & Cirrose & 0 \\
\hline TTO & $\mathbf{m}$ & $\mathbf{b}$ & 2 & Cirrose & $\mathbf{0}$ \\
\hline IC & $\mathbf{m}$ & $\mathbf{b}$ & 36 & Cirrose & $\mathbf{0}$ \\
\hline SLF & $\mathbf{f}$ & $\mathbf{b}$ & 40 & Hepatite & $\mathbf{R}$ \\
\hline ICH & $\mathbf{m}$ & $\mathbf{m}$ & 24 & Hepatite & $\mathbf{R}$ \\
\hline MIM & $\mathbf{m}$ & $\mathbf{b}$ & 27 & Cirrose & $\mathbf{R}$ \\
\hline AFS & $\mathbf{f}$ & $\mathbf{b}$ & 52 & Insuficiência & $\mathbf{R}$ \\
\hline PMC & $\mathbf{m}$ & $\mathbf{b}$ & 59 & Hepatite & 0 \\
\hline ETSS & $\mathbf{m}$ & $\mathbf{b}$ & 5 & Calazar & $\mathbf{O}$ \\
\hline SRL & $\mathbf{m}$ & $\mathbf{p}$ & 33 & Cirrose & 0 \\
\hline ES & $\mathbf{m}$ & $\mathbf{b}$ & 51 & Cirrose & 0 \\
\hline GZ & $\mathbf{m}$ & $\mathbf{b}$ & $\mathbf{5}$ & Cirrose & 0 \\
\hline IVL & $\mathbf{m}$ & $\mathbf{b}$ & 46 & Cirrose & 0 \\
\hline ASD & $\mathbf{f}$ & $\mathbf{p}$ & 44 & Cirrose & 0 \\
\hline S.JA & $\mathbf{m}$ & $\mathbf{b}$ & 60 & Hepatite & $\mathrm{O}$ \\
\hline IPA & $\mathbf{m}$ & $\mathbf{b}$ & 66 & Cirrose & $\mathbf{R}$ \\
\hline $\mathbf{A F}$ & $\mathbf{f}$ & $\mathbf{b}$ & 41 & Cirrose & 0 \\
\hline CAQ & $\mathbf{m}$ & b & 23 & Cirrose & 0 \\
\hline ALP & m & $\mathbf{b}$ & 22 & Cirrose & $\mathbf{R}$ \\
\hline nOH & $\mathbf{f}$ & b & 53 & Hepatite & O \\
\hline RCB & $\mathbf{m}$ & b & 4 & Hepatite & 0 \\
\hline
\end{tabular}

Tabela 1 - Nome, sexo (m, masculino; $f$, feminino), raga (b, branca; $p$, preta; $m$, malata), idade em anas, exceto para o caso MJA no qual é referida em meses (m), patologia hepdtica e evolugão ( $R$, recuperą̧ão; $O$, bbito) dos 50 pacientes. 
Denominamos «recuperação» casos de pacientes que receberam alta hospitalar e «óbito» casos de pacientes que faleceram na vigência de encefalopatia hepśtica. Onze pacientes (22\%) se recuperaram e 39 pacientes (78\%) faleceram. Na tabela 2 apresentamos us diagnósticos das patologias hepáticas, com o número de casos e percentual. Os 50 pacientes apresentaram um total de 63 episódios de coma ou pré-coma hepático. Em 38 foi possível estabelecer os prováveis fatores precipitantes, apresentados na täbela 3.

\begin{tabular}{lcc}
\hline Patologia hepática & No de casos & $\%$ \\
\hline \hline Cirrose hepática & 30 & $60 \%$ \\
Hepatite & 15 & $30 \%$ \\
Esquistossomose & 1 & $2 \%$ \\
Cirrose e esquistossomose & 1 & $2 \%$ \\
Calazar (?) & 1 & $2 \%$ \\
Insuficiência hepática (cirrose?) & 1 & $2 \%$ \\
Sarcoma de células de Küpffer & 1 & $2 \%$ \\
Total & 50 & $100 \%$ \\
\hline
\end{tabular}

Tabela 2 - Diagnostico das patologias hepaticas, com mämero de casos e percentual.

\begin{tabular}{lc}
\hline Fatores precipitantes & No de vezes \\
\hline \hline Hemorragia digestiva & 17 \\
Quadros infecciosos & 11 \\
Desidratação & 5 \\
Procedimentos cirúrgicos & 2 \\
Ingestão protéica & 2 \\
Ingestão de fenotiazina & 1 \\
Total & 38 \\
Sem fatores precipitantes detectáveis & 25 \\
\hline Tabela s - Fratores precipitantes de 38 episodios de encefa- \\
lopatia hepditica.
\end{tabular}

Foram feitas 55 gasometrias arteriais em 34 pacientes; todas mostraram alcalose respiratória. Em 18 exames houve acidose metabólica concomitante. Ers 3 exames houve alcalose mista (tabela 4). Incontramos correlação entre a gravidade do coma $\leftarrow$ o pH sanguíneo (tabela 5). Os 18 exames que mostraram acidose metabólica associada : alcalose respiratória foram feitos em 8 pacientes que tinham outros diagnósticos (tabela 6).

Foram comuns associações de patologias. Assim, alcoolismo foi diagnosticado eir 20 pacientes; desnutriç̃o em 6; tuberculose pulmonar em 5; polineuropatia periférica c pancreatite em 4; pelagra, insuficiência cardiaca e hipertensão em 3; doença de Chagas, 


\begin{tabular}{lc}
\hline & No de exames \\
\hline \hline Alcalose respiratória & 34 \\
Alcalose respiratória e Acidose metabólica & 18 \\
Alcalose respiratória e metabólica & 3 \\
Total & 55 \\
\hline
\end{tabular}

Tabela \& - Resultados de 55 gasometrias arteriais realizadias em 34 pacientes.

\begin{tabular}{rccc}
\hline pH sanguineo & Pré-coma & Coma superficial & Coma profundo \\
\hline $\mathbf{7 , 4 2}-7,46$ & $\mathbf{X X}$ & $\mathbf{X X X}$ & - \\
$\mathbf{7 , 4 7}-7,51$ & $\mathbf{X X}$ & $\mathbf{X X X}$ & $\mathbf{X X}$ \\
$7,52-7,56$ & - & $\mathbf{X X X}$ & $\mathbf{x X X X X}$ \\
\hline
\end{tabular}

Tabela 5 - Relacão entre a gravidade do coma e o pH sanguineo em 20 gasometrias arteriais em que o exame foi feito no momento da avaliaço neturológica. $X=u m a$ gasometria.

\begin{tabular}{lc}
\hline & No de casos \\
\hline Pneumonia & 3 \\
Pielonefrite & 1 \\
Diabetes e insuficiência renal crônica & 1 \\
Pancreatite aguda & 1 \\
Pancreatite e insuficiência renal & 1 \\
Total & 7 \\
\hline
\end{tabular}

Tabela 6 - Outros diagnosticos dos 7 pacientes cujas gasometrias revelanam acidose metabólica associada a alcalose respiratória.

esquistossomose e insuficiência renal crônica em 2; diabetes, anemia falciforme e escorbuto em 1. Autópsia foi realizada em 20 casos: em 2 casos o crânio não foi abertci; em 5, a causa mortis foi: hemorragia digestiva em dois casos e, nos demais, insuficiência renal aguda, pneumonite aguda e trombose de veia porta. Na tabela 7 apresentamos os casos de autópsia em que foi feito estudo macroscópico do cérebro e cuja causa mortis foi coma hepático, relacionando nome, presença ou ausência de edema cerebral e tipo de hérnia. Os tipos de herniacão mais comuns forem: fronto-temporal (4 vezes), amigdalas cerebelares (3 vezes) e uncus (uma vez). 


\begin{tabular}{|c|c|c|c|}
\hline Nome & & $\begin{array}{l}\text { Presença ou ausência } \\
\text { de edema cerebral }\end{array}$ & Tipo de herniação \\
\hline GRP & & + & - \\
\hline MLM & & $\therefore$ & - \\
\hline TFV & & - & - \\
\hline FSA & & + & FT, AC \\
\hline GCS & & + & FT \\
\hline JBE & & + & $\mathbf{A C}$ \\
\hline CMS & & + & $\mathbf{U}, \mathbf{F T}, \mathbf{A C}$ \\
\hline BMN & & + & FT \\
\hline ICS & & - & - \\
\hline GB & & - & - \\
\hline BH & & - & - \\
\hline AMN & & + & - \\
\hline IC & & + & - \\
\hline Total & $\begin{array}{l}\text { com sdema: } 8 \\
\text { sem edema: } 5\end{array}$ & & com herniações: 5 \\
\hline
\end{tabular}

Tabela y - Nome, presenga (+) ou ausencia (-) de edema cerebral e ocorrencia de herniacão cerebral (F'T, fronto-temporal; $A C$, amigdalas cerebelares; $U$, uncus) em 13 casos.

\section{COMENTARIOS}

As alterações iniciais mais freqüentes foram apatia e sonolência. Em alguns casos surgiu delírio agitado. Por vezes houve estabilização do quadro por horas ou dias, com flutuações; outras vezes bouve rápida progressão para estupor e coma. Em alguns casos pudemos constatar agnosia visual, apraxia de construção, disgrafia, e sinais de síndrome frontal, com ecolalia, perseveração de linguagem, reflexos de preensão forçada e persecução obrigatória. Nos casos crônicos, durante remissão, encontramos alterações de personalidade 10,1.t. As alterações motoras mais comuns incluem tremores, asterixis ou "flapping", hipertonia e hiperreflexia profunda. Observamos negativismo motor ou paratonia (das Gegenhalten) em dois casos. Com o aprofundamento do coma podem surgir posturas de decorticação ou descerebração e o sinal de Babinski. As vezes há mioclonias multifocais, nistagmo e sinais motores localizados, que oscilam de uma a outra topografia. Em nossa série apenas um paciente apresentou crise convulsiva $10,12,14$.

Quanto à patogenia, sabe-se que a via normal do alfa-ceto-glutarato para succinil-CoA, no ciclo de Krebs, seria prejudicada pelo aumento da formaçao de ácido glutâmico a partir da aminação redutiva do alfa-ceto-glutarato, e aumento da formação de glutamina a partir de amônia e ácido glutâmico, resultando em alteração na glicólise aeróbica, com perda considerável de moléculas de ATP e redução de energia para o metabolismo celular oxidativo. C aumento de glutamina no líquido cefalorraqueano (LCR), a diminuição do consumo de oxigênio pelo cérebro e o aumento do ácido pirúvico no plasma e LCR durante o coma hepático, serviriam de base para esta hipótese 7,14,18. 
Devido a isto o tratamento clássico, realizado em 49 de nossos pacientes, baseia-se na dieta aprotéica e esterilização da flora intestinal, visando diminuir a produção de amônia ${ }^{13}$. Outros autores defendem o papel da metionina c falsos neurotransmissores, de onde advém o tratamento empírico com clortetraciclina e L-Dopa ${ }^{2,9}$. Recentemente têm sido relatados bons resultados com o emprego da solução balanceada de aminoácidos preconizada por Fischer, que consiste na hiperalimentação parenteral com solução rica em leucina, isoleucina e valina e pobre em tirosina, fenilalanina e triptofano 3,4,5,15. Apenas um de nossos pacientes, com hepatite aguda fulminante, recebeu esse tratamento, já em fase terminal. Evoluiu para óbito.

O eletrencefalograma, realizado em 5 de nossos pacientes, mostrou alentecimento difuso do traçado, às vezes com sofrimento cerebral, principalmente nos casos graves, e ondas trifásicas ${ }^{8}$. Plum e Posner acreditam que a encefalopatia, não acompanhada de alcalose respiratória ou metabólica, não é de origem hepática. De fato, em nossa casuística foram realizadas 55 gasometrias arteriais, e todas mostraram alcalose respiratória. Em 18 exames de 8 pacientes, a alcalose respiratória associou-se a acidose metabólica; entretanto, em 7 destes 8 pacientes outros diagnósticos explicariam o baixo pH sanguíneo (tabela 5). Em três, havia alcalose metabólica concomitante. Assim, o achado de alcalose respiratória, diretamente proporcional à gravidade do coma, é fundamental no estabelecimento do diagnóstico ${ }^{10}$.

As alterações neuropatológicas incluem edema cerebral, presente em 8 de nossos 13 casos examinados. Em 5 havia herniações. Ware e col. relataram 16 casos de edema cerebral em 32 casos estudados, sendo que em 4 havia evidências de herniação ${ }^{17}$. A patogenia é desconhecida; entretanto é possível que contribua a hiperventilação com hipocapnia, vasocontricção cerebral e hipofluxo. Microscopicamente a substância cinzenta do córtex, cerebelo e núcleos da base apresenta aumento no tamanho e número dos astrócitos e comumente encontra-se a alteraçăo tipo 2 de Alzheimer. O núcleo mostra-se túrgido e aquoso, com nucléolo proeminente e silhueta irregular, recortada. Nos casos crônicss há alterações irreversíveis; as camadas profundas podem apresentar necrose laminar e o córtex torna-se adelgaçado com a perda de neurônios e fibras. A participação do cerebelo e núcleos da base explica o aparecimento de sindromes extrapiramidais: Podem ocorrer desmielinizações dos tratos piramidais, que se associam a paraplegia espástica 6,11,16,17. Um de nossos pacientes apresentou hemiparesia espástica esquerda.

\section{RESUMO}

Os autores apresentam estudo retrospectivo de 50 pacientes com encefalopatia hepática atendidos no Hospital das Clínicas da Faculdade de Medicina de Ribeirão Preto a partir de 1959 e discutem os aspectos clínicos, laboratoriais e anátomo-patológicos. Expõem os diagnósticos das hepatopatias, os fatores precipitantes dos episódios de coma e pré-coma hepático e enfatizam o papel da gasometria arterial no estabelecimento do diagnóstico. 


\section{SUMMARY}

Hepatic encephalopathy: a study of 50 cases.

The authors present a retrospective study of 50 patients with hepatic encephalopathy treated at the University Hospital, Faculty of Medicine of Ribeirão Preto since 1959 and discuss clinical, laboratory and anatomo-pathologic aspects. The diagnoses of the different types of liver disease as well as the factors that triggered the episodes of hepatic coma and pre-coma are reported and the role of the measurement of arterial blood gases in establishing the diagnosis is emphasized.

\section{RËFERENCIAS}

1. DAVIDSON, C.S. \& GABUZDA, G.J. - Hepatic coma. In: Schiff, L. (ed.) - Diseases of the Liver. Ed. 4. Lippincott, Philadelphia, 1975.

2. FISCHER, J.E. \& BALDESSARINI, R.J. - False neurotransmitters and hepatic failure. Lancet 2:75, 1971.

3. FISCHER, J.E.; YOSHIMURA, N.; AGUIRRE, A.; JAMES, J.H.; CUMMINGS, M.G.; ABEL, R.M. \& DEINDOERFER, F. - Plasma amino acids in patients with hepatic encephalopathy. Amer. J. Surg. 127:40, 1974.

4 FISCHER, J.E.; FUNOVICS, J.M.; AGUIRRE, A.; JAMES, J.FI.; KEANE, J.M.; WESDORP, R.I.C.; YOSHIMURA, N. \& WESTMAN, T. - The role of plasma amino acids in hepatic encephalopathy. Surgery $78: 276,1975$.

5. FISCHER, J.E.; ROSEN, H.M.; EBEID, A.M.; JAMES, H.; KEANE, J.M. \& SOETERS, P.B. - The effect of normalization of plasma aminc acids on hepatic encephalopathy in man. Surgery 80:77, 1976.

6. HURWITZ, L.J. \& MONTGOMERY, D.A.D. - Persistent choreo-athetosis movements in liver disease. Arch. Neurol. (Chicago) 13:421, 1965.

7. MANGUM, J.; LAMONS, D. \& FRIEDLANDER, W.J. - Neurologic changes in a patient with a portocaval shunt and the relationship to hepatic coma. Amer. J. Med. 21:306, 1956.

8. NIEDERMEYER, E. - Eletroencephalogram: Basic Principles, Clinical Applications and Related Fields. Urban \& Schwartzenberg, Baltimore, 1982.

9 PHEAR. E.A.; RUEBNER, B.; SHERLOCK, S. \& SUMMERSKILL, W.H.J. Methionine toxicity in liver disease and its prevention by chlortetracycline. Clin. Sci. 15:93, 1956.

10. PLUM, F. \& POSNER, J.B. - Diagnóstico de Estupor e Coma. Fd. 2 Guanabara Koogan, Rio de Janeiro, 1977.

11. SCOBIE, B. \& SUMMERSKILL, W.H.J. - Permanent paraplegia with cirrosis. Arch. int. Med. 113:805, 1964.

12. SHERLOCK, S.; SUMMERSKILL, W.H.J.; SHITE, L.P. \& PHEAR, E.A. Portal-systemic encephalopathy. Neurological complication of liver disease. Lancet $2: 453,1954$.

13. SHERLOCK, S.; SUMMERSKILL, W.H.J. \& DAWSON, A.M. - The treatment and prognosis of hepatic coma. Lancet 2:689, 1956.

14. SHERLOCK, S. - Diseases of the Liver and Biliary System. Ed. 3. Blackwell, Oxford, 1975.

15. SMITH, A.; ROSSI-FANELLI, F.: ZIPARO, V.; JAMES, H.; PERELLE, B.A. \& FISCHER, J.E. - Alterations in plasma and CSF amino acids, amine metabolites in hepatic coma. Ann. Surg. 187:343, 1978.

16. VICTOR, M.; ADAMS, R.D. \& COLE, M. - The acquired (non-wilsonian) type of chronic hepatocerebral degeneration. Medicine 44:345, 1965.

17. WARE, A.J.; DAGOSTINO, A.N. \& COMBES, B. - Cerebral edema: a major complication of massive hepatic necrosis. Gastroenterol. 61:877, 1971.

18. ZIEVE, L - Pathogenesis of hepatic coma. Arch. int. Med. 118:211, 1966. 